\title{
On fuzzy $P$-continuous multifunctions
}

\author{
Erdal Ekici
}




\title{
ON FUZZY P-CONTINUOUS MULTIFUNCTIONS
}

\author{
ERDAL EKICI
}

[Received: February 12, 2003]

\begin{abstract}
Aвstract. In this paper, by using the fuzzy property $P$, some characterisations and properties of certain types of upper (lower) fuzzy continuous multifunctions including upper (lower) fuzzy continuous, upper (lower) fuzzy almost continuous, upper (lower) fuzzy $c$-continuous, upper (lower) fuzzy almost $c$-continuous, upper (lower) fuzzy $c^{*}$-continuous, upper (lower) fuzzy $s$-continuous, upper (lower) fuzzy almost $s$-continuous, upper (lower) fuzzy $\ell$-continuous, upper (lower) fuzzy almost $\ell$-continuous functions are given.
\end{abstract}

Mathematics Subject Classification: 54A40, $03 \mathrm{E} 72$

Keywords: Continuity, fuzzy multifunction

\section{InTRODUCTION}

It is well known that several types of fuzzy upper (lower) continuous multifunctions are given in literature. By using property $P$, our main goal here is to give some characterisations and properties of certain types of upper (lower) fuzzy continuous multifunctions including upper (lower) fuzzy continuous, upper (lower) fuzzy almost continuous, upper (lower) fuzzy $c$-continuous, upper (lower) fuzzy almost $c$-continuous, upper (lower) fuzzy $c^{*}$-continuous, upper (lower) fuzzy $s$-continuous, upper (lower) fuzzy almost $s$-continuous, upper (lower) fuzzy $\ell$-continuous, upper (lower) fuzzy almost $\ell$-continuous functions.

Fuzzy sets on a universe $X$ will be denoted by Greek letters as $\mu, \rho, \eta$, etc. Fuzzy points will be denoted by $x_{\varepsilon}, y_{v}$, etc. For any fuzzy points $x_{\varepsilon}$ and any fuzzy set $\mu$, we write $x_{\varepsilon} \in \mu$ iff $\varepsilon \leq \mu(x)$. A fuzzy set $x_{\varepsilon}$ is called quasi-coincident with a fuzzy set $\rho$, denoted by $x_{\varepsilon}$ q $\rho$, iff $\varepsilon+\rho(x)>1$. A fuzzy set $\mu$ is called quasi-coincident with a fuzzy set $\rho$, denoted by $\mu \mathrm{q} \rho$, iff there exists a $x \in X$ such that $\mu(x)+\rho(x)>1$. In this paper we use the concept of a fuzzy topological space as introduced by [1]. By int $(\mu)$, $\operatorname{cl}(\mu)$ and $\operatorname{co}(\mu)$, we mean the interior of $\mu$, the closure of $\mu$, and the complement of $\mu$.

Let $F: X \rightarrow Y$ be a fuzzy multifunction from a fuzzy topological space $X$ to a fuzzy topological space $Y$. For any fuzzy set $\mu \leq X, F^{+}(\mu)$ and $F^{-}(\mu)$ are defined by $F^{+}(\mu)=\{x \in X: F(x) \leq \mu\}, F^{-}(\mu)=\{x \in X: F(x)$ q $\mu\}$. We know that $F^{-}(\operatorname{co}(\beta))=\operatorname{co}\left(F^{+}(\beta)\right)$ for any fuzzy set $\beta \leq Y$ [8]. 


\section{Fuzzy $\boldsymbol{P}$-continuous MUltifunctions}

Definition 1. Let $(X, \tau)$ be a fuzzy topological space and let $\mu \leq X$ be a fuzzy set. Then it is said that

(i) $\mu$ is a fuzzy $P$-set if $\mu$ possesses fuzzy property $P$,

(ii) $\mu$ has fuzzy $P$-complement if $\operatorname{co}(\mu)$ possesses fuzzy property $P$.

The following definition of fuzzy $P$-continuity is considered for fuzzy multisetting from [6] in the classical sense.

Definition 2. Let $F: X \rightarrow Y$ be a fuzzy multifunction from a fuzzy topological space $(X, \tau)$ to a fuzzy topological space $(Y, v)$. Then it is said that $F$ is fuzzy lower (upper) $P$-continuous if for each $x_{\varepsilon} \in X$ and for each fuzzy set $\mu$ having a $P$-complement such that $x_{\varepsilon} \in F^{-}(\mu)\left(x_{\varepsilon} \in F^{+}(\mu)\right)$, there exists an open fuzzy set $\rho$ containing $x_{\varepsilon}$ such that $\rho \leq F^{-}(\mu)\left(\rho \leq F^{+}(\mu)\right)$.

The following table gives us the list of some types of lower (upper) fuzzy $P$ continuous multifunctions with property $P$.

The definitions of lower (upper) fuzzy $c$-continuous, lower (upper) fuzzy almost $c$-continuous, lower (upper) fuzzy $c^{*}$-continuous, lower (upper) fuzzy $s$-continuous, lower (upper) fuzzy $\ell$-continuous and lower (upper) fuzzy almost $\ell$-continuous multifunctions are considered for fuzzy multisetting from [2, 3, 10, 4, 5] and [7], respectively.

The definitions of lower (upper) fuzzy continuous and lower (upper) fuzzy almost continuous multifunctions are considered from [8] and [9], respectively.

$\mathrm{n}^{\circ} \quad$ Fuzzy $P$-set $\quad$ Fuzzy lower $P$-con. Fuzzy upper $P$-con.

1. f. closed

f. l. con. f. u. con.

2. f. regular closed

f. 1. almost con.

f. u. almost con.

3. f. closed compact

f. $1 . c$-con.

f. u. $c$-con.

4. f. regular closed compact

f. 1. almost $c$-con.

f. u. almost $c$-con.

5. f. closed countable compact

6. f. closed connected

f. $1 . c^{*}$-con.

f. u. $c^{*}$-con.

7. f. regular closed connected

f. $1 . s$-con.

f. u. $s$-con.

8. f. closed Lindelöf

f. 1. almost $s$-con.

f. u. almost $s$-con.

9. f. regular closed Lindelöf

f. $1 . \ell$-con.

f. u. $\ell$-con.

f. 1. almost $\ell$-con.

f. u. almost $\ell$-con.

We know that a net $\left(x_{\varepsilon_{\alpha}}^{\alpha}\right)$ in a fuzzy topological space $(X, \tau)$ is said to be eventually in the fuzzy set $\mu \leq X$ if there exists an index $\alpha_{0} \in J$ such that $x_{\varepsilon_{\alpha}}^{\alpha} \in \mu$ for all $\alpha \geq \alpha_{0}$.

The following theorem gives us some characterisation of fuzzy lower (upper) $P$ continuous multifunctions. 
Theorem 1. Let $F: X \rightarrow Y$ be a fuzzy multifunction from a fuzzy topological space $(X, \tau)$ to a fuzzy topological space $(Y, v)$. Then the following statements are equivalent:

(i) $F$ is a lower (upper) fuzzy P-continuous multifunction.

(ii) For each $x_{\varepsilon} \in X$ and for each fuzzy set $\mu$ having a P-complement such that $F\left(x_{\varepsilon}\right)$ q $\mu\left(F\left(x_{\varepsilon}\right) \leq \mu\right)$, there exists an open fuzzy set $\rho$ containing $x_{\varepsilon}$ such that if $y_{\beta} \in \rho$, then $F\left(y_{\beta}\right)$ q $\mu\left(F\left(y_{\beta}\right) \leq \mu\right)$.

(iii) $F^{-}(\mu)\left(F^{+}(\mu)\right)$ is an open fuzzy set for any fuzzy set $\mu \leq Y$ having a $P$ complement.

(iv) $F^{+}(\operatorname{co}(\mu))\left(F^{-}(\operatorname{co}(\mu))\right)$ is a closed fuzzy set for any fuzzy set $\mu \leq Y$ having a $P$-complement.

(v) For each $x_{\varepsilon} \in X$ and for each net $\left(x_{\varepsilon_{\alpha}}^{\alpha}\right)$ which converges to $x_{\varepsilon}$ in $X$ and for each fuzzy set $\rho \leq Y$ having a P-complement such that $x_{\varepsilon} \in F^{-}(\rho)\left(x_{\varepsilon} \in\right.$ $\left.F^{+}(\rho)\right)$, the net $\left(x_{\varepsilon_{\alpha}}^{\alpha}\right)$ is eventually in $F^{-}(\rho)\left(F^{+}(\rho)\right)$.

Proof. (i) $\Leftrightarrow$ (ii). This statement is obvious.

(i) $\Leftrightarrow$ (iii). Let $x_{\varepsilon} \in F^{-}(\mu)$ and let $\mu$ be a fuzzy set having a $P$-complement. From (i), there exists an open fuzzy set $\rho$ containing $x_{\varepsilon}$ such that $\rho \leq F^{-}(\mu)$. It follows that $x_{\varepsilon} \in \operatorname{int}\left(F^{-}(\mu)\right)$ and (iii) holds.

The converse can be shown easily. clear.

(iii) $\Leftrightarrow\left(\right.$ iv). Since $F^{-}(\operatorname{co}(\mu))=\operatorname{co}\left(F^{+}(\mu)\right)$ and $F^{+}(\operatorname{co}(\mu))=\operatorname{co}\left(F^{-}(\mu)\right)$, the proof is

(i) $\Rightarrow(\mathrm{v})$. Let $x_{\varepsilon_{\alpha}}^{\alpha}$ be a net which converges to $x_{\varepsilon}$ in $X$ and let $\rho \leq Y$ be any fuzzy set having a $P$-complement such that $x_{\varepsilon} \in F^{-}(\rho)$. Since $F$ is a lower fuzzy $P$-continuous multifunction, it follows that there exists an open fuzzy set $\mu \leq X$ containing $x_{\varepsilon}$ such that $\mu \leq F^{-}(\rho)$. Since $x_{\varepsilon_{\alpha}}^{\alpha}$ converges to $x_{\varepsilon}$, it follows that there exists an index $\alpha_{0} \in J$ such that $x_{\varepsilon_{\alpha}}^{\alpha} \in \mu$ for all $\alpha \geq \alpha_{0}$. From here, we obtain that $x_{\varepsilon_{\alpha}}^{\alpha} \in \mu \leq F^{-}(\rho)$ for all $\alpha \geq \alpha_{0}$. Thus, the net $\left(x_{\varepsilon_{\alpha}}^{\alpha}\right)$ is eventually in $F^{-}(\rho)$.

(v) $\Rightarrow$ (i). Suppose that (i) is not true. There exists a point $x_{\varepsilon}$ and a fuzzy set $\mu$ having a $P$-complement with $x_{\varepsilon} \in F^{-}(\mu)$ such that $\rho \nless F^{-}(\mu)$ for each open fuzzy set $\rho \leq X$ containing $x_{\varepsilon}$. Let $x_{\varepsilon_{\rho}} \in \rho$ and $x_{\varepsilon_{\rho}} \notin F^{-}(\mu)$ for each open fuzzy set $\rho \leq X$ containing $x_{\varepsilon}$. Then for the neighbourhood net $\left(x_{\varepsilon_{\rho}}\right), x_{\varepsilon_{\rho}} \rightarrow x_{\varepsilon}$, but $\left(x_{\varepsilon_{\rho}}\right)$ is not eventually in $F^{-}(\mu)$. This is a contradiction. Thus, $F$ is a fuzzy lower (upper) $P$-continuous multifunction.

The proof of the fuzzy upper $P$-continuity of $F$ is similar to that given above.

Example. Let $F: X \rightarrow Y$ be a fuzzy multifunction from a fuzzy topological space $(X, \tau)$ to a fuzzy topological space $(Y, v)$. Then the following statements are equivalent to Theorem 1 when we take fuzzy $P$-set as fuzzy closed compact set:

(i) $F$ is a lower (upper) fuzzy $c$-continuous multifunction, 
(ii) For each $x_{\varepsilon} \in X$ and for each fuzzy open set $\mu$ having a compact complement such that $F\left(x_{\varepsilon}\right)$ q $\mu\left(F\left(x_{\varepsilon}\right) \leq \mu\right)$, there exists an open fuzzy set $\rho$ containing $x_{\varepsilon}$ such that if $y_{\beta} \in \rho$, then $F\left(y_{\beta}\right)$ q $\mu\left(F\left(y_{\beta}\right) \leq \mu\right)$,

(iii) $F^{-}(\mu)\left(F^{+}(\mu)\right)$ is an open fuzzy set for any fuzzy open set $\mu \leq Y$ having a compact complement,

(iv) $F^{+}(1-\mu)\left(F^{-}(1-\mu)\right)$ is a closed fuzzy set for any fuzzy open set $\mu \leq Y$ having a compact complement,

(v) For each $x_{\varepsilon} \in X$ and for each net $\left(x_{\varepsilon_{\alpha}}^{\alpha}\right)$ which converges to $x_{\varepsilon}$ in $X$ and for each fuzzy open set $\rho \leq Y$ having a compact complement such that $x_{\varepsilon} \in F^{-}(\rho)$ $\left(x_{\varepsilon} \in F^{+}(\rho)\right)$, the net $\left(x_{\varepsilon_{\alpha}}^{\alpha}\right)$ is eventually in $F^{-}(\rho)\left(F^{+}(\rho)\right)$.

Definition 3. Suppose that $(X, \tau),(Y, v)$ and $(Z, \omega)$ are fuzzy topological spaces. If $F_{1}: X \rightarrow Y$ and $F_{2}: Y \rightarrow Z$ are fuzzy multifunctions, then the fuzzy multifunction $F_{2} \circ F_{1}: X \rightarrow Z$ is defined by $\left(F_{2} \circ F_{1}\right)\left(x_{\varepsilon}\right)=F_{2}\left(F_{1}\left(x_{\varepsilon}\right)\right)$.

Theorem 2. Let $(X, \tau),(Y, v),(Z, \omega)$ be fuzzy topological spaces and let $F: X \rightarrow Y$ and $G: Y \rightarrow Z$ be fuzzy multifunctions. If $F: X \rightarrow Y$ is an upper fuzzy continuous multifunction and $G: Y \rightarrow Z$ is an upper fuzzy $P$-continuous multifunction, then $G \circ F: X \rightarrow Z$ is an upper fuzzy $P$-continuous multifunction.

Proof. Let $\mu \leq Z$ be any fuzzy set having a $P$-complement. From the definition of $G \circ F$, we have $(G \circ F)^{+}(\mu)=F^{+}\left(G^{+}(\mu)\right)$. Since $G$ is an upper fuzzy $P$-continuous multifunction, it follows that $G^{+}(\mu)$ is an open fuzzy set. Since $F$ is an upper fuzzy continuous multifunction, it follows that $F^{+}\left(G^{+}(\mu)\right)$ is an open fuzzy set. This shows that $G \circ F$ is an upper fuzzy $P$-continuous multifunction.

Theorem 3. Let $F: X \rightarrow Y$ be a fuzzy multifunction from a fuzzy topological space $(X, \tau)$ to a fuzzy topological space $(Y, v)$. If $F$ is a lower (upper) fuzzy $P$-continuous multifunction and $\mu \leq X$ is a fuzzy set, then the restriction multifunction $\left.F\right|_{\mu}: \mu \rightarrow Y$ is a lower (upper) fuzzy P-continuous multifunction.

Proof. Suppose that $\beta \leq Y$ is a fuzzy set having a $P$-complement. Let $x_{\varepsilon} \in \mu$ and let $\left.x_{\varepsilon} \in F^{-}\right|_{\mu}(\beta)$. Since $F$ is a lower fuzzy $P$-continuous multifunction, it follows that there exists a fuzzy open set $x_{\varepsilon} \in \rho$ such that $\rho \leq F^{-}(\beta)$. From here we obtain that $x_{\varepsilon} \in \rho \wedge \mu$ and $\rho \wedge \mu \leq\left. F^{-}\right|_{\mu}(\beta)$. Thus, we show that the restriction multifunction $\left.F\right|_{\mu}$ is lower fuzzy $P$-continuous.

The proof of the fuzzy upper $P$-continuity of $\left.F\right|_{\mu}$ is similar to the above.

Theorem 4. Let $f: X \rightarrow Y$ be a fuzzy function from a fuzzy topological space $(X, \tau)$ to a fuzzy topological space $(Y, v)$. Let $\left\{\gamma_{\alpha}: \alpha \in \Phi\right\}$ be an open cover of $X$. If the restriction multifunction $F_{\alpha}=F_{\gamma_{\alpha}}$ is a lower (upper) fuzzy P-continuous multifunction for each $\alpha \in \Phi$, then $F$ is lower (upper) fuzzy P-continuous.

Proof. Let $\mu \leq Y$ be a fuzzy set having a $P$-complement. Since $\mathrm{F}_{\alpha}$ is lower fuzzy $P$-continuous for each $\alpha$, from Theorem $1, F_{\alpha}^{-}(\mu) \leq$ int $_{\gamma_{\alpha}}\left(F_{\alpha}^{-}(\mu)\right)$ and from here 
$F^{-}(\mu) \wedge \gamma_{\alpha} \leq \operatorname{int}_{\gamma_{\alpha}}\left(F^{-1}(\mu) \wedge \gamma_{\alpha}\right)$ and $F^{-}(\mu) \wedge \gamma_{\alpha} \leq \operatorname{int}\left(F^{-1}(\mu)\right) \wedge \gamma_{\alpha}$. Since $\left\{\gamma_{\alpha}: \alpha \in \Phi\right\}$ is an open cover of $X$, it follows that $F^{-}(\mu) \leq \operatorname{int}\left(F^{-}(\mu)\right.$ ). Thus from Theorem 1, we obtain that $F$ is a lower fuzzy $P$-continuous multifunction.

The proof of the fuzzy upper $P$-continuity of $F$ is similar to the above.

Definition 4. Suppose that $F: X \rightarrow Y$ is a fuzzy multifunction from a fuzzy topological space $X$ to a fuzzy topological space $Y$. The fuzzy graph multifunction $G_{F}: X \rightarrow X \times Y$ of $F$ is defined as $G_{F}\left(x_{\varepsilon}\right)=\left\{x_{\varepsilon}\right\} \times F\left(x_{\varepsilon}\right)[8]$.

Theorem 5. Suppose that a finite product of fuzzy $P$-sets is a fuzzy $P$-set and $X$ is a fuzzy $P$-set. Let $F: X \rightarrow Y$ be a fuzzy multifunction from a fuzzy topological space $(X, \tau)$ to a fuzzy topological space $(Y, v)$. If the graph function of $F$ is lower (upper) fuzzy $P$-continuous, then $F$ is a lower (upper) fuzzy P-continuous multifunction.

Proof. For the fuzzy sets $\beta \leq X, \eta \leq Y$, we take

$$
(\beta \times \eta)(z, y)= \begin{cases}0 & \text { if } z \notin \beta \\ \eta(y) & \text { if } z \in \beta .\end{cases}
$$

Let $x_{\varepsilon} \in X$ and let $\mu \leq Y$ be a fuzzy set having a $P$-complement such that $x_{\varepsilon} \in$ $F^{-}(\mu)$. We obtain that $x_{\varepsilon} \in G_{F}^{-}(X \times \mu)$. Since fuzzy graph multifunction $G_{F}$ is fuzzy lower $P$-continuous and $X$ is a fuzzy $P$-set, it follows that there exists an open fuzzy set $\rho \leq X$ containing $x_{\varepsilon}$ such that $\rho \leq G_{F}^{-}(X \times \mu)$. From here, we obtain that $\rho \leq F^{-}(\mu)$. Thus, $F$ is a fuzzy lower $P$-continuous multifunction.

The proof of the fuzzy upper $P$-continuity of $F$ is similar to the above.

Theorem 6. Suppose that $(X, \tau)$ and $\left(X_{\alpha}, \tau_{\alpha}\right)$ are fuzzy topological spaces and $X_{\alpha}$ is a fuzzy $P$-set where $\alpha \in J$. Let $F: X \rightarrow \prod_{\alpha \in J} X_{\alpha}$ be a multifunction from $X$ to the product space $\prod_{\alpha \in J} X_{\alpha}$ and let $P_{\alpha}: \prod_{\alpha \in J} X_{\alpha} \rightarrow X_{\alpha}$ be the projection multifunction for each $\alpha \in J$ which is defined by $P_{\alpha}\left(\left(x_{\alpha}\right)\right)=\left\{x_{\alpha}\right\}$ and let a product of fuzzy $P$-sets be a fuzzy $P$-set. If $F$ is a fuzzy upper $P$-continuous multifunction, then $P_{\alpha} \circ F$ is a fuzzy upper P-continuous multifunction for each $\alpha \in J$.

Proof. Take any $\alpha_{0} \in J$. Let $x_{\varepsilon} \in X$ and let $\mu_{\alpha_{0}} \leq X_{\alpha_{0}}$ be a fuzzy set having a $P$ complement such that $x_{\varepsilon} \in\left(P_{\alpha_{0}} \circ F\right)^{+}\left(\mu_{\alpha_{0}}\right)$. We know that $x_{\varepsilon} \in\left(P_{\alpha_{0}} \circ F\right)^{+}\left(\mu_{\alpha_{0}}\right)=$ $F^{+}\left(P_{\alpha_{0}}^{+}\left(\mu_{\alpha_{0}}\right)\right)=F^{+}\left(\mu_{\alpha_{0}} \times \prod_{\alpha \neq \alpha_{0}} X_{\alpha}\right)$. Since $F$ is a fuzzy upper $P$-continuous multifunction and a product of fuzzy $P$-sets is a fuzzy $P$-set, it follows that there exists an open fuzzy set $\rho \leq X$ containing $x_{\varepsilon}$ such that $\rho \leq F^{+}\left(\mu_{\alpha_{0}} \times \prod_{\alpha \neq \alpha_{0}} X_{\alpha}\right)=$ $F^{+}\left(P_{\alpha_{0}}^{+}\left(\mu_{\alpha_{0}}\right)\right)=\left(P_{\alpha_{0}} \circ F\right)^{+}\left(\mu_{\alpha_{0}}\right)$. This shows that $P_{\alpha_{0}} \circ F$ is a fuzzy upper $P$-continuous multifunction.

Thus we obtain that $P_{\alpha} \circ F$ is a fuzzy upper $P$-continuous multifunction for each $\alpha \in J$.

Theorem 7. Suppose that for each $\alpha \in J,\left(X_{\alpha}, \tau_{\alpha}\right),\left(Y_{\alpha}, v_{\alpha}\right)$ are fuzzy topological spaces and $Y_{\alpha}$ possesses property $P$ for each $\alpha \in J$. Let $F_{\alpha}: X_{\alpha} \rightarrow Y_{\alpha}$ be a 
multifunction for each $\alpha \in J$ and let $F: \prod_{\alpha \in J} X_{\alpha} \rightarrow \prod_{\alpha \in J} Y_{\alpha}$ be defined by $F\left(\left(x_{\alpha}\right)\right)=$ $\prod_{\alpha \in J} F_{\alpha}\left(x_{\alpha}\right)$ from the product space $\prod_{\alpha \in J} X_{\alpha}$ to the product space $\prod_{\alpha \in J} Y_{\alpha}$. If $F$ is an upper fuzzy $P$-continuous multifunction and the product of fuzzy $P$-sets is a fuzzy $P$-set, then each $F_{\alpha}$ is an upper fuzzy $P$-continuous multifunction for each $\alpha \in J$.

Proof. Let $\mu_{\alpha} \leq Y_{\alpha}$ be a fuzzy set having a $P$-complement. Then $\mu_{\alpha} \times \prod_{\alpha \neq \beta} Y_{\beta}$ is a fuzzy set having a $P$-complement. Since $F$ is an upper fuzzy $P$-continuous multifunction, it follows that $F^{+}\left(\mu_{\alpha} \times \prod_{\alpha \neq \beta} Y_{\beta}\right)=F^{+}\left(\mu_{\alpha}\right) \times \prod_{\alpha \neq \beta} X_{\beta}$ is an open fuzzy set. Consequently, we obtain that $F^{+}\left(\mu_{\alpha}\right)$ is an open fuzzy set. Thus, we show that $F_{\alpha}$ is an upper fuzzy $P$-continuous multifunction.

Theorem 8. Let $F: X \rightarrow Y$ be a fuzzy function from a fuzzy topological space $(X, \tau)$ to a fuzzy topological space $(Y, v)$. Suppose that $Y$ has a base of neighbourhood such that the complement of each fuzzy set of the base of neighbourhood is the finite union of $P$-sets. If $F$ is lower fuzzy P-continuous multifunction, then $F$ is lower fuzzy continuous.

Proof. Let $x_{\varepsilon} \in X$ and let $\mu$ be any fuzzy open set such that $x_{\varepsilon} \in F^{-}(\mu)$. Then there exists a fuzzy point $y_{v} \in \mu$ such that $x_{\varepsilon} \in F^{-}\left(y_{v}\right)$. Since $Y$ has a base of neighbourhood such that the complement of each fuzzy set of the base of neighbourhood is finite unions of $P$-sets, it follows that there exists a neighbourhood $\beta$ of $y_{v}$ such that $\beta \leq \mu$ and $\operatorname{co}(\beta)=\bigvee_{i=1}^{n} \eta_{i}$ where each $\eta_{i}$ is a $P$-set. From here we obtain that $\beta=\bigwedge_{i=1}^{n} \operatorname{co}\left(\eta_{i}\right)$. Then $y_{v} \in \operatorname{co}\left(\eta_{i}\right)$ and $x_{\varepsilon} \in F^{-}\left(\operatorname{co}\left(\eta_{i}\right)\right)$ for each $i=1,2, \ldots, n$. Since $F$ is a lower fuzzy $P$-continuous multifunction, it follows that there exists an open set $\rho_{i}$ containing $x_{\varepsilon}$ such that $\rho_{i} \leq F^{-}\left(\operatorname{co}\left(\eta_{i}\right)\right)$. We take $\rho=\bigwedge_{i=1}^{n} \rho_{i}$, then $\rho=\bigwedge_{i=1}^{n} \rho_{i} \leq \bigwedge_{i=1}^{n} F^{-}\left(\operatorname{co}\left(\eta_{i}\right)\right)=F^{-}\left(\bigwedge_{i=1}^{n} \operatorname{co}\left(\eta_{i}\right)\right)=F^{-}(\beta) \leq F^{-}(\mu)$. Thus, we obtain that $F$ is a lower fuzzy $P$-continuous multifunction.

Theorem 9. Suppose that $\left(X_{1}, \tau_{1}\right),\left(X_{2}, \tau_{2}\right),\left(Y_{1}, v_{1}\right)$ and $\left(Y_{2}, v_{2}\right)$ are fuzzy topological spaces and $F_{1}: X_{1} \rightarrow Y_{1}, F_{2}: X_{2} \rightarrow Y_{2}$ are fuzzy multifunctions and suppose that $\eta \times \beta$ is a fuzzy $P$-set iff $\eta$ and $\beta$ are fuzzy $P$-sets for any fuzzy sets $\eta \leq Y_{1}, \beta \leq Y_{2}$. Let $F_{1} \times F_{2}: X_{1} \times X_{2} \rightarrow Y_{1} \times Y_{2}$ be a fuzzy multifunction which is defined by $\left(F_{1} \times F_{2}\right)\left(x_{\varepsilon}, y_{v}\right)=F_{1}\left(x_{\varepsilon}\right) \times F_{2}\left(y_{v}\right)$. Then $F_{1} \times F_{2}$ is an upper fuzzy $P$-continuous multifunction iff $F_{1}$ and $F_{2}$ are upper fuzzy $P$-continuous multifunctions.

Proof. We know that $\left(\mu^{*} \times \beta^{*}\right)\left(x_{\varepsilon}, y_{v}\right)=\min \left\{\left(\mu^{*}(x), \beta^{*}(y)\right\}\right.$ for any fuzzy sets $\mu^{*}, \beta^{*}$ and for any fuzzy points $x_{\varepsilon}, y_{v}$.

Let $\mu \times \beta \leq Y_{1} \times Y_{2}$ be a fuzzy set having a $P$-complement. We know that $\left(F_{1} \times\right.$ $\left.F_{2}\right)^{+}(\mu \times \beta)=F_{1}^{+}(\mu) \times F_{2}^{+}(\beta)$ and $\rho \times \xi$ is a fuzzy $P$-set iff $\rho$ and $\xi$ are fuzzy $P$-sets for any fuzzy sets $\rho \leq Y_{1}, \xi \leq Y_{2}$. From Theorem 1, the proof is obtained.

Theorem 10. Suppose that $(X, \tau),(Y, v),(Z, \omega)$ are fuzzy topological spaces and $F_{1}$ : $X \rightarrow Y, F_{2}: X \rightarrow Z$ are fuzzy multifunctions and suppose that $\eta \times \beta$ is a fuzzy $P$-set iff $\eta$ and $\beta$ are fuzy $P$-sets for any fuzzy sets $\eta \leq Y, \beta \leq Z$. Let $F_{1} \times F_{2}: X \rightarrow Y \times Z$ be a fuzzy multifunction which is defined by $\left(F_{1} \times F_{2}\right)\left(x_{\varepsilon}\right)=F_{1}\left(x_{\varepsilon}\right) \times F_{2}\left(x_{\varepsilon}\right)$. Then 
$F_{1} \times F_{2}$ is an upper fuzzy $P$-continuous multifunction iff $F_{1}$ and $F_{2}$ are upper fuzzy $P$-continuous multifunctions.

Proof. $(\Rightarrow)$ Let $x_{\varepsilon} \in X$ and let $\mu \leq Y, \beta \leq Z$ be fuzzy sets having $P$-complements such that $x_{\varepsilon} \in F_{1}^{+}(\mu)$ and $x_{\varepsilon} \in F_{2}^{+}(\beta)$. Then we obtain that $F_{1}\left(x_{\varepsilon}\right) \leq \mu$ and $F_{2}\left(x_{\varepsilon}\right) \leq \beta$ and from here, $F_{1}\left(x_{\varepsilon}\right) \times F_{2}\left(x_{\varepsilon}\right)=\left(F_{1} \times F_{2}\right)\left(x_{\varepsilon}\right) \leq \mu \times \beta$. We have $x_{\varepsilon} \in\left(F_{1} \times F_{2}\right)^{+}(\mu \times \beta)$. Since $F_{1} \times F_{2}$ is an upper fuzzy $P$-continuous multifunction, it follows that there exists an open fuzzy set $\rho$ containing $x_{\varepsilon}$ such that $\rho \leq\left(F_{1} \times F_{2}\right)^{+}(\mu \times \beta)$. We obtain that $\rho \leq F_{1}^{+}(\mu)$ and $\rho \leq F_{2}^{+}(\beta)$. Thus we obtain that $F_{1}$ and $F_{2}$ are upper fuzzy $P$-continuous multifunctions.

$(\Leftarrow)$ Let $x_{\varepsilon} \in X$ and let $\mu \times \rho \leq Y \times Z$ be a fuzzy set having a $P$-complement such that $x_{\varepsilon} \in\left(F_{1} \times F_{2}\right)^{+}(\mu \times \rho)$. We obtain that $\left(F_{1} \times F_{2}\right)\left(x_{\varepsilon}\right)=F_{1}\left(x_{\varepsilon}\right) \times F_{2}\left(x_{\varepsilon}\right) \leq \mu \times \rho$. From here we have $F_{1}\left(x_{\varepsilon}\right) \leq \mu$ and $F_{2}\left(x_{\varepsilon}\right) \leq \rho$ and then $x_{\varepsilon} \in F_{1}^{+}(\mu)$ and $x_{\varepsilon} \in F_{2}^{+}(\rho)$. Since $F_{1}$ and $F_{2}$ are upper fuzzy $P$-continuous multifunctions, it follows that there exist fuzzy open sets $\eta$ containing $x_{\varepsilon}$ and $\beta$ containing $x_{\varepsilon}$ such that $\eta \leq F_{1}^{+}(\mu)$ and $\beta \leq F_{2}^{+}(\rho)$. We take $x_{\varepsilon} \in \eta \wedge \beta$. Then we obtain that $\eta \wedge \beta \leq F_{1}^{+}(\mu)$ and $\eta \wedge \beta \leq F_{2}^{+}(\rho)$. Hence we have $\eta \wedge \beta \leq\left(F_{1} \times F_{2}\right)^{+}(\mu \times \rho)$. Thus, we show that $F_{1} \times F_{2}$ is an upper fuzzy $P$-continuous multifunction.

\section{REFERENCES}

[1] Chang, C. L.: Fuzzy topological spaces, J. Math. Appl., 24 (1968), 182-190.

[2] Gentry, K. R. and Hoyle, H. B.: c-continuous functions, Yokohama Math. J., 18, (1970), 71-76.

[3] Hwang, S. H.: Almost c-continuous functions, J. Korean Math. Soc., 14 (1978), 229-234.

[4] Khedr, F. H. and Elbaki, S. A. A.: Fuzzy s-continuous functions and semilocally connected fuzzy topological spaces, Fuzzy Sets and Systems, 70 (1995), 113-117.

[5] Конц, J. K.: A class of mappings containing all continuous mappings, Glasnik Matematicki, 16 (1981), 361-368.

[6] Kонц, J. K.: A unified approach to continuous and certain non-continuous functions, Symposium on General Topology and its Applications, Delhi (1978). A unified view of (complete) regularity and certain variants of complete regularity, Canad. J. Math., 36 (1984), 783-794.

[7] Konstadilaki-Savvopoulou, Ch. and Reilly, I. L.: Almost $\ell$-continuous functions, Glasnik Matematicki, 25 (1990), no. 2, 363-370.

[8] MukherJee, M. N. ANd Malakar, S.: On almost continuous and weakly continuous fuzzy multifunctions, Fuzzy Sets and Systems, 41 (1991), 113-125.

[9] Papageorgiou, N. S.: Fuzzy topology and fuzzy multifunctions, J. Math. Anal. Appl., 109 (1985), 397-425.

[10] PARK, Y. S.: c $c^{*}$-continuous functions, J. Korean Math. Soc., 8 (1971), 69-72.

Author's Address

\section{Erdal Ekici:}

Department of Mathematics, Cumhuriyet University, Sivas 58140, Turkey

E-mail address: eekici@cumhuriyet.edu.tr 